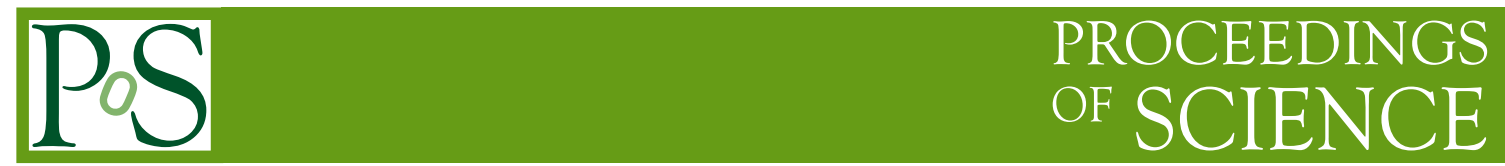

\title{
Novae and other Cataclysmic Variables
}

\author{
T.J. O'Brien* \\ Jodrell Bank Centre for Astrophysics, School of Physics \& Astronomy, The University of \\ Manchester, Oxford Road, Manchester M13 9PL, UK \\ E-mail: tim.obrien@manchester.ac.uk
}

\begin{abstract}
I review recent work on radio observations of novae and other cataclysmic variables (CVs). I pay particular attention to the multiwavelength campaign on the 2006 outburst of RS Ophiuchi, and to recent work on the dwarf nova SS Cyg. I discuss how these observations are providing evidence for the ejection of radio jets from white dwarfs during outbursts in some CVs. Rapidresponse, high-resolution, radio detection and imaging that can be provided with e-VLBI clearly has an important future role, particularly in the area of determining the geometry of radio emission during outbursts of CVs.
\end{abstract}

Science and Technology of Long Baseline Real-Time Interferometry:

The 8th International e-VLBI Workshop, EXPReSO9

June 22 - 262009

Madrid, Spain

${ }^{*}$ Speaker. 


\section{Cataclysmic Variables}

Cataclysmic variables (CVs; [1]) are interacting binary stars comprising a white dwarf (WD) in orbit around another star, typically a main-sequence dwarf but occasionally a red giant or other evolved star. Matter is transferred from the companion onto the surface of the WD, in most cases via an accretion disc around the WD. CVs exhibit a wide range of behaviour characterised by variations in visual brightness on timescales of seconds to hours to weeks to years. They are divided into several classes.

Nova outbursts result from a thermonuclear explosion in the hydrogen-rich material accreted onto the surface of the WD ([2]; Fig. 1). The explosion does not destroy the WD and can recur (after accretion resumes) on timescales ranging from around ten years in the recurrent novae to, it is thought, thousands of years in the classical novae. The explosion leads to the ejection of $10^{-8}-10^{-4} \mathrm{M}_{\odot}$ from the WD at speeds of several hundred to several thousand $\mathrm{km} \mathrm{s}^{-1}$. Novae brighten by around 6-13 magnitudes in only a few days and then fade back to quiescence over periods ranging from several weeks to years.

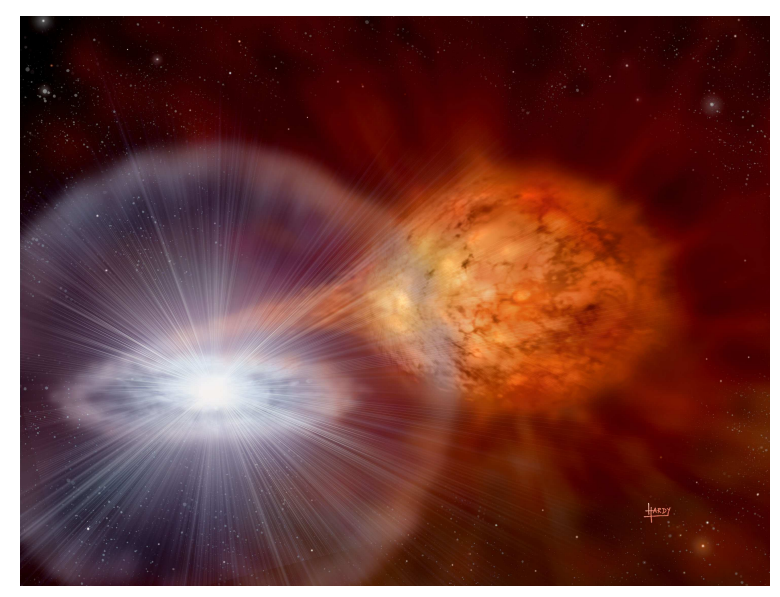

Figure 1: Artist's impression of the recurrent nova RS Oph showing the explosion on the white dwarf which is accreting material from a companion red giant star (David A. Hardy/AstroArt \& PPARC).

As the name implies the dwarf novae have outbursts which are, typically, not quite as spectacular as the classical or recurrent novae. The brightness changes by factors of a few to a hundred or so, but the outbursts are repeated much more often, on timescales of days to years. It is thought that dwarf nova outbursts are the result of changes in the mass transfer rate through the accretion disc.

About $25 \%$ of the WDs in CV systems have detectable magnetic fields which can disrupt the accretion flow. When the WD magnetic field is stronger than about $10 \mathrm{MG}$ then the accretion disc cannot form and the gas from the companion star is channelled via the field directly onto the poles of the WD. The radiation from the accreting gas in the strong magnetic field is cyclotron emission and is strongly polarized. These systems are known as polars, or AM Her stars after the prototype of the class. In polars the WD is locked to the companion via the interaction of their magnetic fields so that it spins on its axis with the same period as the orbital period. If the field is relatively weak then the system may not be locked and these are known as asynchronous polars. WDs with 
fields less than a few million gauss are not magnetically locked and typically rotate with much shorter periods then the binary. Only the inner parts of the disc are disrupted in the region where the dynamics of the flow is dominated by the magnetic energy density. These systems are known as intermediate polars or sometimes DQ Her stars. Few IP's have detectable polarization as the polarized component is swamped by emission from the disc.

Other interacting binaries which overlap in classification with the CVs are the symbiotic stars [3]. Characterised by evidence in the spectrum for both a hot and a cool star, they consist of a red giant in orbit around, in most cases, a white dwarf although some accretors are main-sequence stars, hot sub-dwarfs or neutron stars. A wide range of behaviour is exhibited including nova-like outbursts (e.g. the recurrent nova RS Oph, see later, is sometimes classed as a symbiotic) and the ejection of jets.

\section{Radio Emission from CV's}

Several surveys of radio emission from CV's and X-ray binaries ([4], [5], [6], [7]) indicate that there are no persistent radio sources amongst the non-magnetic CV's.

However, at least 18 classical novae have been detected in outburst [8]. In this case the emission is free-free produced by the photoionised ejected shell at a temperature of around $10^{4} \mathrm{~K}$. The radio emission appears rather later than the visual peak, at first optically thick and rising as the shell expands, then fading as the ejecta become optically thin and their density declines. Radio interferometric observations have been used to track the evolution of the ejecta in six cases [9]: GK Per [10], QU Vul [11], V1974 Cyg [12], V705 Cas [13], V723 Cas [14] and V1494 Aq1 [15]. The radio and optical imaging reveal clumpy, spherical or ellipsoidal structures.

In contrast there have been only a few reports of radio emission from dwarf novae in outburst - SU UMa [16] and EM Cyg [17] have been detected only a few times. The most recent detection is of SS Cyg [18], see later.

Of the ten known recurrent novae only RS Ophiuchi has been detected in outburst in the radio [19]. RS Oph is discussed in more detail in the next section. Recently, however, a suspected new recurrent nova V2672 Oph was detected with VLA [20] with indications that it shows similar behaviour to RS Oph.

There have also been a number of surveys of the magnetic CV's (e.g. [21], [22], [23]), most recently using the VLA to investigate all those within $100 \mathrm{pc}$ [24]. These have shown that only three appear to be persistent radio sources: AR UMa, AM Her, AE Aqr (and also the pre-CV V471 Tau). There have been sporadic detections of flares in a few others: V834 Cen, ST LMi, DQ Her, and BG CMi.

As [24] point out, non-magnetic CVs are not radio-loud (except in some cases in outburst). This might suggest that a magnetic WD is a necessary condition for radio emission. However, isolated magnetic WDs are not radio sources so it is clearly not the magnetic WD alone that explains magnetic $\mathrm{CV}$ radio emission. Persistent emission has not been detected in any CV with an accretion disc outside of outburst, so maybe the disc somehow prevents radio emission. Possible models require a magnetized secondary to also be present and radio emission then arises from gyrosynchrotron in the combined magnetosphere. However, there is still insufficient data from an 
inhomogeneous sample of sources and progress probably requires higher-sensitivity, co-ordinated observations expected to be provided by upcoming instrumentation.

\section{A few recent sources of interest}

\subsection{RS Oph}

RS Oph is a recurrent nova which has been seen to undergo outbursts every 10-20 years or so, the most recent in 2006 [25]. Since the ejection from the WD takes place into the wind of a red giant companion, a shock system is set up which leads to copious soft X-ray emission and a combination of thermal and non-thermal radio emission.

A major multi-wavelength campaign of observations followed the 2006 outburst. High-cadence monitoring of the X-ray emission with the Swift satellite showing evidence of emission from the expanding shock and from continued thermonuclear burning on the WD. In the radio [26], the Multi-Element Radio-Linked Interferometer Network (MERLIN) and the Very Large Array (VLA) were used to detect the nova only four days after discovery and follow its light curve as it brightened to a C-band flux density of around $50 \mathrm{mJy}$ before fading, peaking again 40 days after outburst, and then fading away over the following months.

Radio imaging with MERLIN, the Very Long Baseline Array (VLBA) and the European VLBI Network (EVN), resolved, for the first time, the expanding synchrotron-emitting shock wave [19] (Fig. 2a). This gradually broke up into several components extended east-west (Fig. 2b) suggesting either shaping by an asymmetric red giant wind and/or jet ejection from the WD. VLA images at $43 \mathrm{GHz}$ [27] reveal that the extended radio lobes appear to be driven by highly collimated outflows with half-opening angles of just a few degrees, suggesting that the WD in RS Oph does indeed launch jets following outburst.
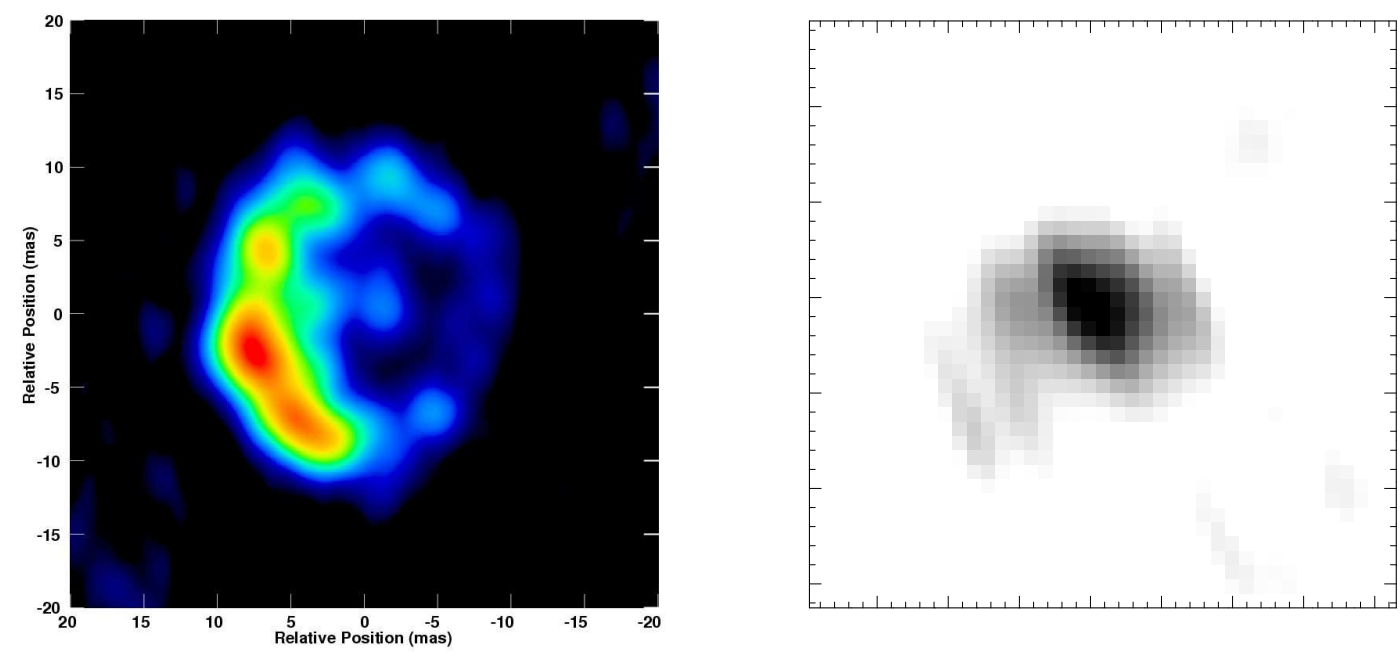

Figure 2: (a) At left, VLBA image of RS Oph at 5 GHz from 2006 Feb 26, 14 days after outburst [19]. Peak flux density is $4.7 \mathrm{mJy}^{-1}$ beam ${ }^{-1}$ and the resolution is 3.3 mas. (b) At right, MERLIN image of RS Oph at $6 \mathrm{GHz}$ from 2006 May 9, 86 days after outburst; major tickmarks are 0.1 arcsec apart.

The energetic outbursts and short recurrence time of RS Oph, suggest that the WD is very close to the Chandrasekhar limit. This makes RS Oph and other similar objects candidates for progenitors 
of Type Ia supernovae. Another fast recurrent nova which is expected to undergo an outburst in the near future is U Sco. This is the fastest-known nova indicating a massive WD and therefore also a potential Type Ia SN progenitor. It brightens by 10 magnitudes in just a few hours and then fades by 3 magnitudes over the next few days. Expansion velocities are around $10,000 \mathrm{~km} \mathrm{~s}^{-1}$ and there has been the suggestion of jet ejection in the optical line emission. A multi-wavelength monitoring campaign coordinated by Schaefer, Pagnotta and Schlegel is already underway in preparation for the outburst and, although the secondary in U Sco is a sub-giant star rather than a red giant and hence a strong external shock system is less likely to develop, rapid-response radio observations could prove interesting.

\subsection{SS Cyg}

Interpreting the behaviour of the accreting WDs in CVs by analogy with accreting neutron stars or black holes in X-ray binaries (XRBs), [18] construct a disc-fraction luminosity diagram which can be compared to the X-ray hardness-intensity diagrams for XRBs. In the latter, radio emission appears in the form of jets when the X-ray source is hard and brightening, suggesting that radio emission from dwarf novae might be seen when on the rise at the beginning of an outburst. However this phase only lasts around 24 hours making it hard to catch.

SS Cyg is a prototypical dwarf nova. Discovered in 1896, it brightens by around four magnitudes approximately every 4-10 weeks. In April 2007 [18] used reports from the American Association of Variable Star Observers (AAVSO) that SS Cyg had gone into outburst to trigger VLA and MERLIN observations. Slightly after the beginning of the visual outburst, the source was detected at $8.5 \mathrm{GHz}$, rising sharply to a flux density of $1.1 \mathrm{mJy}$, before fading rapidly and then more slowly over the course of the next 3 weeks. The source was unresolved with MERLIN at L-band indicating a size less than 0.2 arcsec. A number of arguments suggest the source to be synchrotron emission. The geometry is hard to determine but [18] argue in favour of jets with a launching efficiency $\sim 10 \%$, making dwarf novae very interesting jet laboratories part-way between young stellar objects and XRB's. They are continuing to monitor several other dwarf novae using the same approach.

\section{Summary}

Over recent years there has been progress in understanding the origin of persistent radio emission in CVs, but arguably the most exciting work relates to the accumulation of evidence for the ejection of jets in some CVs and that, in cases where the WD is massive, some may eventually explode as Type Ia supernovae.

As recent work on RS Oph and SS Cyg in particular has shown, new insights can be obtained with co-ordinated rapid-response observations. Since significant evolution takes place on timescales of a day or less, these observations are challenging. Rapid-response high-resolution radio detection and imaging that can be provided with e-VLBI clearly has an important future role, particularly in the area of determining the geometry of radio emission during outbursts of CVs

\section{References}

[1] Warner, B., 1995, Cataclysmic Variable Stars, Cambridge University Press 
[2] Bode, M.F., Evans, A., 2008, eds. Classical Novae, Cambridge University Press

[3] Kenyon, S.J., Webbink, R.F., 1984, ApJ, 279, 252

[4] Cordova, F.A., Hjellming, R.M., Mason, K.O., 1983, PASP, 95, 69

[5] Fuerst, E., Benz, A., Hirth, W., Geffert, M., Kiplinger, A., 1986, A\&A, 154, 377

[6] Echevarria, J., 1987, Ap\&SS, 130,103

[7] Nelson, R.F., Spencer, R.E., 1988, MNRAS, 234, 1105

[8] Seaquist, E.R., Bode, M.F., 2008, in Classical Novae, Cambridge University Press, p141

[9] O’Brien, T.J., Bode, M.F., 2008, in Classical Novae, Cambridge University Press, p285

[10] Reynolds, S.P., Chevalier, R.A., 1984, ApJ, 281, L33

[11] Taylor, A.R., Hjellming, R.M., Seaquist, E.R., Gehrz, R.D., 1988, Nature, 335, 235

[12] Eyres, S.P.S., Davis, R.J., Bode, M.F., 1996, MNRAS, 279, 249

[13] Eyres, S.P.S., Bode, M.F., O’Brien, T.J., Watson, S.K., Davis, R.J., 2000, MNRAS, 318, 1086

[14] Heywood, I., O’Brien, T.J., Eyres, S.P.S., Bode, M.F., Davis, R.J., 2005, MNRAS, 362, 469

[15] Eyres, S.P.S., Heywood, I., O’Brien, T.J., Ivison, R.J., Muxlow, T.W.B., Elkin, V.G., 2005, MNRAS, 358,1019

[16] Benz, A.O., Fuerst, E., Kiplinger, A.L., 1983, Nature, 302, 45

[17] Benz, A.O., Güdel, M., 1989, A\&A, 218, 137

[18] Körding, E., Rupen, M., Knigge, C., Fender, R., Dhawan, V., Templeton, M., Muxlow, T.W.B., 2008, Science, 320, 1318

[19] O’Brien, T.J., Bode, M.F., Porcas, R.W., Muxlow, T.W.B., Eyres, S.P.S., Beswick, R.J., Garrington, S.T., Davis, R.J., Evans, A., 2006, Nature, 442, 279

[20] Krauss Hartman, M.I., Rupen, M.P., Mioduszewski, A.J., 2009, Astronomer's Telegram, 2195

[21] Chanmugam, G., Dulk, G.A., ApJ, 255, L107

[22] Beasley, A.J., Bastian, T.S., Ball, Lewis, Wu, K., 1994, AJ, 108, 2207

[23] Mason, P.A., Fisher, P.L., Chanmugam, G., 1996, A\&A, 310, 132

[24] Mason, P.A., Gray, C.L., 2007, ApJ, 660, 662

[25] Evans, A, Bode, M.F., O’Brien, T.J., Darnley, M.J., 2008, RS Ophiuchi (2006) and the Recurrent Nova Phenomenon, ASP Conf. Ser. Vol. 401

[26] Eyres, S.P.S., O’Brien, T.J., Beswick, R., Muxlow, T.W.B., Anupama, G.C., Kantharia, N.G., Bode, M.F., Gawroński, M.P., Feiler, R., Evans, A., Rushton, M.T., Davis, R.J., Prabhu, T., Porcas, R., Hassall, B.J.M., 2009, MNRAS, 395, 1533

[27] Sokoloski, J.L., Rupen, M.P., Mioduszewski, A.J., 2008, ApJ, 685, L137 